\title{
CLASS PRESERVING AUTOMORPHISMS OF BLACKBURN GROUPS
}

\author{
ALLEN HERMAN ${ }^{\oplus}$ and YUANLIN LI
}

(Received 21 July 2004; revised 5 October 2005)

Communicated by E. A. O'Brien

\begin{abstract}
In this article, a Blackburn group refers to a finite non-Dedekind group for which the intersection of all nonnormal subgroups is not the trivial subgroup. By completing the arguments of M. Hertweck, we show that all conjugacy class preserving automorphisms of Blackburn groups are inner automorphisms.
\end{abstract}

2000 Mathematics subject classification: primary 20D45; secondary 16 S34.

\section{Introduction}

If every subgroup of a group is a normal subgroup, then it is called a Dedekind group. For a group $G$ that is not a Dedekind group, let $R(G)$ denote the intersection of all nonnormal subgroups of $G$. Finite non-Dedekind groups $G$ with the property that $R(G)>1$ were classified by Blackburn in [1], and so we will refer to these as Blackburn groups. Recent interest in Blackburn groups has arisen in the theory of integral group rings because these groups were shown to satisfy the normalizer property over the integers [5]. This means that the normalizer of $G$ in the unit group of $\mathbb{Z} G$ is as small as possible - the product of $G$ with the central units of $\mathbb{Z} G$. This property, or the lack of it, has implications to some important questions for integral group rings, including the isomorphism problem and the Zassenhaus conjectures.

The arguments in [5] left open the question of whether or not inner automorphisms of Blackburn groups are the only ones that preserve conjugacy classes, from which the

This research was supported in part by Discovery Grants from the Natural Sciences and Engineering Research Council of Canada.

(C) 2006 Australian Mathematical Society 1446-7887/06 $\$$ A2.00+0.00 
normalizer property (over any integral domain in which the group order is not invertible) would follow. In light of well-known examples of finite metabelian groups that possess non-inner class preserving automorphisms (see, for example, [4, page 250]), the possibility that such a group could be a Blackburn group cannot be immediately ruled out. Nevertheless, in this article we show in Theorem 3.2 that class preserving automorphisms of Blackburn groups must be inner automorphisms. Our approach is to complete the argument for this given in [2, Proposition 22.4], which is based on an incomplete description of Blackburn groups. In the final section, we address a similar issue in the argument for [2, Theorem 23.8], which gives an application of Theorem 3.2 that characterizes a certain type of unit in a group ring of a torsion group.

\section{Blackburn's classification}

A group $G$ is a $Q$-group if $G$ has an abelian subgroup $A$ of index 2 which is not elementary abelian, and $G=\langle A, b\rangle$ for some $b \in G \backslash A$ of order 4 with $x^{b}=x^{-1}$ for all $x \in A$. If, in addition, there is an $a \in A$ with $a^{2}=b^{2}$, then $G$ is said to be a $Q^{*}$-group. Fix the notation so that

$$
Q_{2^{n}}=\left\langle s, t: s^{2^{n-1}}=1, t^{2}=s^{2^{n-2}}, s^{t}=s^{-1}\right\rangle
$$

denotes the generalized quaternion group of order $2^{n}$ for $n \geq 3$. Also, $E_{2}$ denotes a finite elementary abelian 2-group.

We now give Blackburn's classification [1]. If a Blackburn group $G$ is a $p$-group for some prime $p$, then $p=2$ and one of the following holds:

(1) $G \cong Q_{8} \times C_{4} \times E_{2}$.

(2) $G \cong Q_{8} \times Q_{8} \times E_{2}$.

(3) $G$ is a $Q$-group.

If a Blackburn group $G$ is not of prime power order, then one of the following holds:

(a) $G$ has an abelian subgroup $B$ of exponent $k p^{n}$ where $n \geq 1, p$ is a prime, and $(k, p)=1 . G / B$ is cyclic of order $p^{r}$ and if $B u$ generates $G / B, u$ can be chosen so that $u^{p^{\prime}}$ has order $p^{n}$. There exists an integer $\xi \equiv 1 \bmod p^{n}$ such that $x^{u}=x^{\xi}$ for all $x$ in $B$.

(b) $G$ is the direct product of an abelian group of odd order and a 2-group of type (1) or (2) above.

(c) $G$ has a subgroup $H$ of the kind described in (a) with $p=2$ and $r=1 . H$ is of index 2 and if $G$ is generated by $H$ and $t, t$ can be chosen so that $u^{t}=u^{-1}, t^{2}=u^{2^{n}}$, and there exists an $\eta \equiv-1 \bmod 2^{n}$ such that $x^{t}=x^{\eta}$ for all $x$ in $B$.

(d) $G$ has an abelian subgroup $B$ of index 2. $G$ is generated by $B$ and $t$ where $t^{2}$ is 
an element of $B$ of order 2. There exists a $\zeta \equiv-1 \bmod 2^{n}$ such that $x^{t}=x^{\zeta}$ for all $x$ in $B$.

(e) $G$ is the direct product of $H$, a quaternion group of order 8 , and an elementary abelian 2-group, where $H$ is of odd order and is of the kind described in (a).

In [2, Theorem 22.2], Hertweck describes Blackburn groups in the same way, except that types (a) and (c) are presented differently:

$\left(\mathrm{a}^{\prime}\right) \quad G=N \rtimes\langle b\rangle$ with a $p$-element $b$ and an abelian $p^{\prime}$-group $N$. There is (an) $m \in \mathbb{N}$ such that $x^{b}=x^{m}$ for all $x \in N$, and $1 \neq C_{\langle b\rangle}(N) \neq\langle b\rangle$.

(c') $G \cong N \rtimes Q_{2^{n}}$ with $N$ abelian of odd order, and $x^{s}=x^{-1}, x^{t}=x$ for all $x \in N$.

Unfortunately, this means that some Blackburn groups of types (a), (c), and (e) are not covered by Hertweck's condition. The omitted groups of type (a) and (e) are not crucial because these have a normal abelian subgroup with cyclic quotient, and therefore every class preserving automorphism of these groups is forced to be an inner automorphism by [2, Proposition 14.4]. However, the following example of a Blackburn group of type (c) cannot be handled using the arguments in [2]. Let

$$
G \cong\left\langle a, s, t: a^{60}=1, a^{s}=a^{49}, a^{t}=a^{11},\langle s, t\rangle \cong Q_{16}\right\rangle .
$$

This group is a Blackburn group, satisfying the conditions of type (c) in Blackburn's classification, with $R(G)=\left\langle s^{4}\right\rangle \cong C_{2}$. However, $G$ does not satisfy Hertweck's conditions. This example can be generalized to many other such examples, all of the form $(N \times B) \rtimes Q_{2^{n}}$, with $N$ an abelian group of odd order not equal to a prime power, and $B$ an abelian 2-group with $4 \leq \exp (B) \leq 2^{n-2}$. These do not satisfy Hertweck's description when $s$ and $t$ neither fix nor invert all of $N$.

\section{The main result}

Let $\operatorname{Aut}(G)$ be the group of automorphisms of a group $G, \operatorname{Inn}(G)$ its subgroup of inner automorphisms, and $\operatorname{Aut}_{c}(G)$ the subgroup of class preserving automorphisms which leave every conjugacy class of $G$ invariant. Recall that a power automorphism of a group $G$ is an automorphism of $G$ that leaves every subgroup invariant, and thus maps every element of $G$ to a power of itself. We denote the subgroup of power automorphisms of $G$ by $P \operatorname{Aut}(G)$. A universal automorphism of a group $G$ is a power automorphism that maps every element of $G$ to the same power. The subgroup of universal automorphisms of $G$ is denoted $U \operatorname{Aut}(G)$. $U \operatorname{Aut}(G)$ is often trivial when $G$ is not abelian, however, when $G$ is abelian, $U$ Aut $(G)$ is isomorphic to the group of units modulo $\exp (G)$.

LEMMA 3.1. Let $G$ be a finite group. Suppose $A$ is an abelian normal subgroup of $G$, and suppose the restriction of every inner automorphism of $G$ to $A$ is a power 
automorphism of $A$. Then the restriction of every class preserving automorphism of $G$ to $A$ is a universal automorphism of $A$. In particular, if $G$ is abelian, then $P \operatorname{Aut}(G)=U \operatorname{Aut}(G)$.

PROOF. Let $\sigma \in \operatorname{Aut}_{c}(G)$. Let $a$ be an element of $A$ of maximal order. The assumptions imply that there exists an $n \in \mathbb{N}$ such that $\sigma(a)=a^{n}$. Let $B$ be a complement to $\langle a\rangle$ in $A$, so that $A=\langle a\rangle \times B$ and $\exp (B)$ divides $o(a)$. Let $m, \ell \in \mathbb{N}$ be such that $\sigma(b)=b^{m}$ and $\sigma(a b)=(a b)^{\ell}$. Then $a^{\ell} b^{\ell}=\sigma(a b)=\sigma(a) \sigma(b)=a^{n} b^{m}$, and hence $a^{n-\ell}=b^{\ell-m}=1$. Since $o(b)$ divides $o(a), n \equiv \ell \bmod o(a)$ implies that $n \equiv \ell \bmod o(b)$, and so $\sigma(b)=b^{m}=b^{n}$. Therefore, $\sigma(x)=x^{n}$, for all $x \in A$.

It follows from the above argument that every power automorphism of $A$ is a universal automorphism of $A$, and thus the last assertion follows by taking $G=A$.

This brings us to the main result.

THEOREM 3.2. Class preserving automorphisms of Blackburn groups are inner automorphisms.

PROOF. If $G$ is a Blackburn group of type (a) or (d), or if $G$ is a 2-group that is a $Q$-group, then the conclusion is immediate from [2, Proposition 14.4]. Since $\operatorname{Aut}_{c}(H \times K)=\operatorname{Aut}_{c}(H) \times \operatorname{Aut}_{c}(K)$ holds for all groups $H$ and $K$, it follows from the fact that $\operatorname{Aut}_{c}\left(Q_{8}\right)=\operatorname{Inn}\left(Q_{8}\right)$ that $\operatorname{Aut}_{c}(G)=\operatorname{Inn}(G)$ holds for Blackburn groups of type (b) or (e). Similar reasoning can be applied if $G$ is a 2-group isomorphic to either of the types $Q_{8} \times C_{4} \times E_{2}$ or $Q_{8} \times Q_{8} \times E_{2}$. This leaves only Blackburn groups of type (c) to be considered.

Let $G$ be a Blackburn group of type (c). Write $G=A \rtimes Q_{2^{n+1}}$, with $A=N \times B, N$ an abelian normal subgroup of $G$ of odd order, and $B$ an abelian normal 2-subgroup of exponent dividing $2^{n}$. Let

$$
\left\langle u, t: u^{2^{n}}=t^{4}=1, u^{2^{n-1}}=t^{2}, u^{t}=u^{-1}\right\rangle=Q_{2^{n+1}},
$$

and let $\xi \equiv 1 \bmod 2^{n}, \eta \equiv-1 \bmod 2^{n}$ be positive integers such that $a^{u}=a^{\xi}$, $a^{u^{2}}=a$, and $a^{t}=a^{\eta}$ for all $a \in A$. Every element of $G$ can be uniquely written in the form $a u^{i} t^{j}$ for $a \in A, i \in\left\{0, \ldots, 2^{n}-1\right\}$, and $j \in\{0,1\}$. We may assume that $u$ and $t$ act differently and non-trivially on $A$, for otherwise $G$ has a normal abelian subgroup of index 2, and [2, Proposition 14.4] applies.

Let $\sigma \in \operatorname{Aut}_{c}(G)$. By modifying $\sigma$ by an inner automorphism, we may assume $\sigma(t)=t$, and $\sigma(u)=u^{c}=c^{\xi-1} u$, for some $c \in A$. We may assume that $c$ has odd order because $u$ fixes the Sylow 2-subgroup of $A$. By Lemma 3.1, there exists a $\delta \in\{1, \xi, \eta, \xi \eta\}$ such that $\sigma(a)=a^{\delta}$ for all $a \in A$. So $\sigma$ is defined by

$$
\sigma\left(a u^{i} t^{j}\right)=a^{\delta}\left(c^{\xi-1} u\right)^{i} t^{j}=a^{\delta} c^{\xi^{i}-1} u^{i} t^{j} .
$$


By expanding and comparing both sides of

$$
\sigma\left(\left(a u^{i} t^{j}\right)\left(b u^{k} t^{\ell}\right)\right)=\sigma\left(a u^{i} t^{j}\right) \sigma\left(b u^{k} t^{\ell}\right)
$$

for $a, b \in A$, one can show that a necessary condition for any such map defined by the parameters $\delta$ and $c$ to be an automorphism of $G$ is for the condition $(\eta-1)(\xi-1) \equiv 0$ $\bmod o(c)$ to hold.

Since $A$ is abelian, the map $a \mapsto a^{\xi-1}$ defines a group endomorphism on $A$, with image $[A, u]$ and kernel $C_{A}(u)$. Since $u$ centralizes $B$, we have that $[A, u] \subseteq N$. It follows that $a \mapsto a^{(\xi-1)(\eta-1)}$ is a homomorphism from $A$ to $N$, with image [[A, $\left.\left.u\right], t\right]$. Since $u, t$ act coprimely on $N$, we have by Fitting's Lemma [3, Chapter III.13.4] that

$$
A=B \times C_{N}(u) \times[A, u]=B \times C_{N}(u) \times C_{[A, u]}(t) \times[[A, u], t] .
$$

Therefore, every element of odd order in the kernel of the map $a \mapsto a^{(\xi-1)(\eta-1)}$ must lie in $C_{N}(u) C_{N}(t)$. Therefore, if $c=b d$ for some $b \in C_{N}(u), d \in C_{N}(t)$, we can replace $\sigma$ by $\operatorname{conj}\left(d^{-1}\right) \circ \sigma$ to ensure there exists a $\delta \in\{1, \xi, \eta, \xi \eta\}$ such that

$$
\sigma\left(a u^{i} t^{j}\right)=a^{\delta} u^{i} t^{j}
$$

for all $a \in A$ and $u^{i} t^{j} \in Q_{2^{n+1}}$. So it suffices to show that, in order for such an automorphism to be class preserving, $\delta$ must be 1 .

To do this, we will show that whenever $\delta \neq 1$, there exists an $a \in A$ such that at least one of the conjugacy classes of $a u$, at, or aut is not preserved by $\sigma$. For all $a, b \in A, u^{i} t^{j} \in Q_{2^{n+1}}$, we have

$$
\begin{aligned}
(a u)^{b u^{i} t^{\prime}} & =a^{\xi^{i} \eta^{j}} b^{\xi^{i} \eta^{j}(\xi-1)} u^{(-1)^{j}}, \\
(a t)^{b u^{i} t^{\prime}} & =a^{\xi^{i} \eta^{j}} b^{\xi^{i} \eta^{j}(\eta-1)} u^{(-1)^{j}(-2 i)} t, \quad \text { and } \\
(a u t)^{b u^{i} t^{j}} & =a^{\xi^{i} \eta^{j}} b^{\xi^{i} \eta^{j}(\xi \eta-1)} u^{(-1)^{j}(1-2 i)} t .
\end{aligned}
$$

For a typical element $(a u)^{b u^{i} r^{j}}$ in the conjugacy class of $a u, b$ may be chosen from $N$ because $B$ centralizes $u$. If $\delta \in\{\eta, \xi \eta\}$ and if there exists an $a \in A$ such that $a^{u}=a$ and $a^{t} \neq a$, then $\sigma(a u)=a^{\eta} u$. In order for this to be equal to $(a u)^{b u^{i} t^{j}}$ for some $b \in N$, we must have $u=u^{(-1)^{j}}$, so $j$ has to be even. The equation $\sigma(a u)=(a u)^{b u^{i} s^{j}}$ thus reduces to $1 \neq a^{\eta-1}=b^{\xi-1}$. Conjugating both sides by $u$ results in $b^{\xi-1}=a^{\eta-1}=b^{1-\xi}$, and thus $b^{2(\xi-1)}=1$. Since $b$ has odd order, this implies $b^{\xi-1}=1=a^{\eta-1}$, a contradiction.

Now suppose $a^{u} \neq a$ for all $a \in A$. Then $a^{u}=a^{-1}$ for all $a \in A$, and $B$ is elementary abelian and central. If $\delta=\eta$, then choose $a \in N$ so that $a^{t}=a^{-1}$, which we can do because $t$ acts non-trivially on $A$. Then $\sigma(a u t)=a^{-1} u t$ would have to equal $(a u t)^{b u^{\prime} t^{\prime}}$ for some $b \in N$. This forces $u=u^{(-1)^{\prime}(1-2 i)}$. If $j$ is even, then 
$u^{-2 i}=1$, so $i$ is also even, and the equation reduces to $1 \neq a^{-2}=b^{\xi \eta-1}$. Conjugating both sides by $u t$ results in a contradiction as before. If $j$ is odd, then $u^{2}=u^{2 i}$, so $i$ is odd, and the equation reduces to $1 \neq a^{-2}=b^{1-\xi \eta}$. We again reach a contradiction by conjugating both sides by $u t$. If $\delta=\xi \eta$, then choose $a \in N$ so that $a^{t}=a$, which can be done because $u$ and $t$ act differently on $A$. In this case, $\sigma(a t)=a^{-1} t=(a t)^{b u^{\prime} t^{\prime}}$ implies $u^{(-1)^{\prime}(-2 i)}=1$, so $i$ is even, and we are reduced to $1 \neq a^{-2}=b^{\eta^{j}(\eta-1)}$ for some $b \in N$. Since $\eta$ has order 2 modulo the order of $b$, conjugating both sides by $t$ results in a contradiction as before.

Finally, suppose $\delta=\xi$. Again, since $u$ and $t$ act non-trivially and differently on $A$, we can arrange that, by replacing $t$ by $u t$ if necessary (that is, if $a^{t}=a^{-1}$ for all $a \in A$ ), there exists an $a \in A$ such that $a^{u} \neq a$ and $a^{t}=a$. Furthermore, since $u$ centralizes $B$, such an $a$ has odd order. The equation

$$
\sigma(a t)=a^{\xi} t=\sigma(a t)=(a t)^{b u^{i} t^{\prime}}
$$

thus implies that $u^{(-2 i)}=1$, so $i$ must be even, and so we have $1 \neq a^{\xi-1}=b^{\eta^{j}(\eta-1)}$. Since $a$ has odd order, there exists a solution to this equation for which $b$ also has odd order. Again by conjugating both sides by $t$, we obtain $b^{\eta^{j}(\eta-1)}=a^{\xi-1}=b^{\eta^{j}(1-\eta)}$, and we reach a contradiction once again.

Therefore, the only way $\sigma$ can be class preserving is for $\delta=1$. But this implies that we can modify $\sigma$ by inner automorphisms and reach the identity, so $\sigma$ is an inner automorphism.

It should be noted that the conclusion of [2, Proposition 22.4] is that for all Blackburn groups $G, R(G) \leq Z(G)$ and $\operatorname{Aut}_{c}(G)=\operatorname{Inn}(G)$. It is straightforward to see that $R(G)$ is central in $G$ for all Blackburn groups $G$ from Blackburn's classification.

\section{An application to units}

Throughout this section, $G$ denotes a (not necessarily finite) torsion group, $R$ denotes a $G$-adapted ring (an integral domain of characteristic zero in which the order of any element of $G$ is not invertible in $R$ ), and $\mathscr{U}$ denotes the group of units of $R G$. We consider $\mathbb{Z} G$ as a subring of $R G$. An element $y \in R G$ is unipotent if $y-1$ is nilpotent, that is, $(y-1)^{n}=0$ for some positive integer $n$. We seek a characterization of the units of $R G$ that commute with all unipotent elements of $\mathbb{Z} G$. As we mentioned earlier in the introduction, the following result appears as [2, Theorem 23.8], but the proof given there is also based on the incomplete description of Blackburn groups. We will complete Hertweck's proof by accounting for the groups that were missing.

THEOREM 4.1. Assume that $G$ is a non-Dedekind torsion group, and that some $u \in N_{\mathscr{U}}(G)$ commutes with all unipotent elements of $\mathbb{Z} G$. Then $u \in Z(\mathscr{U}) G$. 
ProOF. By [2, Proposition 23.5], we have $[G, u] \leq R(G)$, so we may assume $R(G) \neq 1$. If $w \in \operatorname{supp}(u)$, then $M=R(G)\left\langle\operatorname{supp}\left(u w^{-1}\right)\right\rangle\langle w\rangle$ is a finite normal subgroup of $G$ containing $\operatorname{supp}(u)$. By Hertweck's results, it suffices to consider the case where $M$ is a Blackburn group of type (a) or (e).

Let $M$ be a Blackburn group of type (a) or (e), with $|R(M)|$ a power of the prime $p$. Let $b$ be an element of $M$ of maximal $p$-power order such that $R(M)=\left\langle b^{p^{r}}\right\rangle$. Note that $R(G) \leq R(M)$ (see [1, Lemma $1(\mathrm{a})]$ ), and $R(M) \leq Z(M)$, so $[G, u] \leq Z(M) \leq$ $Z\left(O_{p}(M)\right)$. For every $g \in G$ there is a positive integer $m$ such that $[g, u]=b^{m p^{r}}$. If $b^{p^{\prime}}$ has order $p^{k}$, then it follows that $g^{-1} u^{p^{k}} g=\left(b^{m p^{\prime}} u\right)^{p^{k}}=u^{p^{k}}$, so $u$ has $p$-power order modulo $Z(\mathscr{U})$. By [2, Lemma 19.4], there exists an $x$ in the support of $u$ and a subgroup $P$ of $p^{\prime}$-index in $G$ such that $\left[P, u x^{-1}\right]=1$. Since this implies $a^{x}=a^{u}$ for all $a \in P$, by replacing $u$ by some power equivalent to $u \bmod Z(\mathscr{U})$ if necessary, we can find $y \in\langle x\rangle$ of order a power of $p$ such that $\left[P, u y^{-1}\right]=1$.

We claim that $\operatorname{conj}\left(u y^{-1}\right)$ has order a power of $p$. To see this, note that since $[u, y] \in R(G), u$ and $y$ commute modulo $R(G)$. If $o(y)=p^{c}$, then modulo $R(G)$ we have $\left(u y^{-1}\right)^{p^{c}} \equiv u^{p^{c}}\left(y^{-1}\right)^{p^{c}} \equiv u^{p^{c}}$. Therefore, there exists a $b_{1} \in R(G)$ such that $\left(u y^{-1}\right)^{p^{r}}=u^{p^{c}} b_{1}$. Since $R(G) \leq R(M) \leq Z(M), \operatorname{supp}(u) \leq M$ and $R(M)$ is a $p$-group, $u$ commutes with $b_{1}$ and $o\left(b_{1}\right)=p^{d}$ for some $d \geq 0$. Thus $\left(u y^{-1}\right)^{p^{c+d}}=$ $u^{p^{c+d}} b_{1}^{p^{d}}=u^{p^{c+d}}$. Since $u$ has $p$-power order modulo $Z(\mathscr{U})$, we can choose a large enough integer $e \geq c+d$ such that $\left(u y^{-1}\right)^{p^{e}} \in Z(\mathscr{U})$. It follows that conj $\left(u y^{-1}\right)$ has order a power of $p$, as claimed.

If $S$ is a Sylow $p$-subgroup of $M$, then since $P$ is of $p^{\prime}$-index in $G$, there is a fixed point in the action of $S$ on the set of left cosets of $P$ in $G$, and so there is a coset $g P$ of $P$ such that $s g P=g P$ for all $s \in S$. Therefore, $S^{g} \leq P$, and it follows that $\left[S^{g}, u y^{-1}\right]=1$. In particular, $\operatorname{conj}\left(u y^{-1}\right)$ acts as the identity on $Z\left(O_{p}(M)\right)$. Therefore, we have that $\operatorname{conj}\left(u y^{-1}\right)$ is an automorphism of $R G$ of $p$-power order, which acts as the identity on $G / Z\left(O_{p}(M)\right)$ and $Z\left(O_{p}(M)\right)$, and fixes a subgroup of $p^{\prime}$ index elementwise. By the standard restriction-corestriction argument in 1cohomology (see [3, Chapter I.16.18]), it follows that $\operatorname{conj}\left(u y^{-1}\right)=\operatorname{conj}(h)$ for some $h \in Z\left(O_{p}(M)\right)$. Therefore, $u=z h y$ for some $z \in Z(\mathscr{U})$, so we are done.

\section{References}

[1] N. Blackburn, 'Finite groups in which the nonnormal subgroups have nontrivial intersection', $J$. Algebra 3 (1966), 30-37.

[2] M. Hertweck, Contributions to the integral representation theory of groups (Habilitationsschrift, University of Stuttgart, 2004), available at

http: //elib.uni-stuttgart.de/opus/volltexte/2004/1638/

[3] B. Huppert, Endliche Gruppen I (Springer, New York, 1967). 
[4] S. Jackowski and Z. Marciniak, 'Group automorphisms inducing the identity map on cohomology', J. Pure Appl. Algebra 44 (1987), 241-250.

[5] Y. Li, M. M. Parmenter and S. K. Sehgal, 'On the normalizer property for integral group rings', Comm. Algebra 27 (1999), 4217-4223.

Department of Mathematics and Statistics

University of Regina

Regina

Saskatchewan S4S 0A2

Canada

e-mail: aherman@math.uregina.ca
Department of Mathematics

Brock University

St. Catharine's

Ontario L2S 3A1

Canada

e-mail: yli@brocku.ca 